\title{
Comparison of three HDV-RNA quantitative commercially available tests in untreated and in myrcludex-B treated patients with hdv related chronic hepatitis in a real-life setting
}

APASL Single Topic Conference Delta Hepatitis

June 27-28, 2019 Baku, Azerbaijan

Alessandro Loglio ${ }^{1}$

${ }^{1}$ Italy, ale.loglio@gmail.com

Background and Aim. As new anti-hepatitis delta (HDV) therapies are being developed, highly sensitive and reliable quantitative tests are needed. Aim of the study was to compare three commercially available HDV-RNA kits. Methods. 92 serum samples from 4 categories of patients were tested for HDV-RNA by 3 assays: RoboGene (HDV-RNA quantification 2.0, Aj-Roboscreen, Germany, LLQ $6 \mathrm{IU} / \mathrm{mL}$ ), EurobioPlex (HDV qRT-PCR, Eurobio, France, $100 \mathrm{IU} / \mathrm{mL}$ ) and Dia.Pro (HDV-RNA Quantitation, Dia.Pro Diagnostic Bioprobes, Italy, $50 \mathrm{IU} / \mathrm{mL}$ ). Total RNA was extracted by EZ1 DSP Virus Kit (Qiagen, Hilden, Germany). Results. Group 1: 48 Caucasians with known active HDV-hepatitis [50 years, 56\% males, $73 \%$ cirrhotics, $64 \%$ on tenofovir/entecavir, $80 \%$ undetectable HBV-DNA, 50\% previously interferon-exposed, ALT 66 (24-304) U/L] had a median HDV-RNA of 5.5 (1.1-7.1), 5.9 (08.4), 3.9 (0-6.6) log IU/mL by RoboGene, EurobioPlex and Dia.Pro; viremia tested undetectable in $0(0 \%), 3(6 \%)$ and $5(10 \%)$ patients, respectively. Group 2: $15 \mathrm{HBsAg}$ positive patients [age $42,27 \%$ cirrhotics, $7 \%$ HBeAg-positive, $40 \%$ on tenofovir/entecavir, $73 \%$ undetectable HBV-DNA, 3 with abnormal ALT] had HDV-RNA undetectable with all 3 assays except for one subject who had HDVRNA 697 IU/mL with Dia.Pro. Group 3 included 9 international quality control sera. The only true-negative sample tested negative by all the 3 assays, while the 8 positive controls were correctly identified in $100 \%$, $87.5 \%$ and $25 \%$ of the cases by different assays. Group 4: 21 sera collected during Myrcludex B-treatment. First patient: baseline HDV-RNA was 23,600, 640,006, 12,283 $\mathrm{IU} / \mathrm{mL}$; during therapy, RNA progressively declined with both RoboGene and EurobioPlex, till undetectability at week 36; Dia.Pro gave 5 false-negative results. Second patient: baseline HDV-RNA 392,000, 4,248,001, 1,140 IU/mL; RNA declined with the first two assays but not with the last one ( 2 false-negative results). Overall, the HDVRNA sensitivity was $100 \%$, 92\%, $80 \%$, respectively. Conclusions. RoboGene is the most sensitive and reliable test for HDV-RNA quantification.

"Published on behalf of the Azerbaijan Gastroenterology and Hepatology Association for the APASL STC Hepatitis Delta. All rights reserved. (C) The Author 2019. For permissions please email: editor@ejcs.org or uptodate.az@gmail.com 